\title{
Effects of Desloratadine Citrate Disodium on Serum Immune Function Indices, Inflammatory Factors and Chemokines in Patients with Chronic Urticaria
}

\author{
Juan Wang, Yiding Zhao and Xiaoning Yan
}

\begin{abstract}
Objective: To determine the effects of desloratadine citrate disodium on serum immunological function indices (IgE, C3 and C4), inflammatory factors (IL-4, IL-17 and IL-33), and chemokines (MCP-1, RANTES and Eotaxin) in patients with chronic urticaria.

Study Design: An experimental study.

Place and Duration of Study: Department of Dermatology, Shaanxi Provincial Hospital of Traditional Chinese Medicine, Xi'an, China, from January 2017 to January 2018.

Methodology: A total of 128 patients with chronic urticaria were randomly divided into a control group and an observation group, with 64 patients in each group. The patients in the control group were treated with loratadine and those in the observation group were treated with desloratadine citrate disodium. After 4 weeks of treatment, the serum levels of immune function indices (IgE, C3 and C4), inflammatory factors (IL-4, IL-17 and IL-33), and chemokines (MCP-1, RANTES and Eotaxin) were determined.

Results: After 4 weeks of treatment, the levels of serum IgE, IL-4, IL-17, IL-33, MCP-1, RANTES and Eotaxin in the observation group were lower than those in the control group (all $p<0.001$ ). The levels of serum C3 and C4 in the observation group were higher than those in the control group (both $p<0.001$ ).

Conclusion: Desloratadine citrate disodium can effectively reduce the severity of chronic urticaria, and its therapeutic mechanism may be related to balancing Th1/Th2 cell function, regulating inflammatory mediators and inhibiting the action of chemokines.
\end{abstract}

Key Words: Desloratadine citrate disodium, Chronic urticarial, Immune function, Inflammatory factor, Chemokine.

\section{INTRODUCTION}

Chronic urticaria is a recurrent allergic disease of the skin and mucous membranes, usually lasting for more than six weeks. It mainly manifests as local swelling or itching on the face or limbs, erythema and wheal. Its itching is extremely difficult to bear, which often occurs several times a day and causes great distress for the patients. ${ }^{1,2}$ The cause of chronic urticaria is complex, and for some patients it is related to autoimmunity, but the specific mechanism has not yet been fully defined. 3,4 A second-generation antihistamines turned loratadine is one of the most commonly used medicines for clinical treatment of chronic urticaria.5,6 Desloratadine citrate disodium is the latest antihistamine agent. Pharmacological pharmacokinetic studies have shown that it has higher solubility than other second-generation antihistamines with better safety, so it may have better efficacy for the treatment of chronic urticaria.7,8

Department of Dermatology, Shaanxi Provincial Hospital of

Traditional Chinese Medicine, Xi'an, 710003, China

Correspondence: Dr. Xiaoning Yan, Department of Dermatology,

Shaanxi Provincial Hospital of Traditional Chinese Medicine,

$X{ }^{\prime}$ an, 710003, China

E-mail:rowl26@163.com

Received: June 06, 2018; Accepted: October 12, 2018
Currently, there are few studies on the effects of desloratadine citrate disodium on serum immunological function indices (IgE, C3 and C4), inflammatory factors (IL-4, IL-17 and IL-33), and chemokines (MCP-1, RANTES and Eotaxin) in patients with chronic urticaria.

The objective of this study was to determine effects of desloratadine citrate disodium on serum immunological function indices (IgE, C3 and C4), inflammatory factors (IL-4, IL-17 and IL-33), and chemokines (MCP-1, RANTES and Eotaxin) in patients with chronic urticaria, in order to provide reference for clinical treatment of chronic urticaria.

\section{METHODOLOGY}

This study was conducted at the Department of Dermatology, Shaanxi Provincial Hospital of Traditional Chinese Medicine, Xi'an, China, from January 2017 to January 2018. A total of 128 patients with chronic urticaria treated in our hospital from January 2017 to January 2018 were selected as study subjects. Inclusion criteria were clinically diagnosed as chronic urticaria, with symptoms and signs of urticaria 24 hours before the experiment; and no other antihistamines, anticholinergic agents, receptor agonists, aspirin drugs or glucocorticoids taken within 2 weeks. Exclusion criteria were pregnant women, lactating women, women of childbearing age who planned to have children in the near future; 
those allergic to any ingredient in desloratadine citrate disodium and its preparations; those with severe respiratory, circulatory, digestive, endocrine, hematological, immune, neurological or psychiatric diseases and any other disease that may disturb the experiment results or diseases that affected the safety of the subject; and those with other types of urticaria, such as artificial urticaria, cholinergic urticaria, drug allergy or hereditary angioedema. The study was conducted after approval from the Hospital Ethical and Research Committee. The patients were randomly divided into a control group and an observation group, with 64 patients in each group. Patients in the control group were treated with loratadine, orally, $10 \mathrm{mg} / \mathrm{time}$, once daily for 4 weeks. Patients in the observation group were treated with desloratadine citrate disodium, orally, $8.8 \mathrm{mg} /$ time, once daily for 4 weeks.

After four weeks of treatment, 3-mL fasting cubital venous blood was taken from both groups of patients in the morning, low-speed centrifugated after adding anticoagulant, and the supernatant was collected and stored frozen in a medical deep-freezer for use. The levels of serum immunoglobulin $\mathrm{E}(\mathrm{IgE})$ and complement $\mathrm{C} 3$ and C4 were measured by a fully automated quantitative immunoassay system in both groups. Serum inflammatory cytokines IL-4, IL-17, IL-33 and monocyte/ chemokine-1 (MCP-1), normal $\mathrm{T}$ cell expression and secretion factor (RANTES) and chemotactic factor for eosinophils (Eotaxin) were determined by enzymelinked immunosorbent assay (ELISA).

SPSS version 25.0 software was utilised for statistical data analysis, measurement data shown in Mean $\pm S D$, examined by independent sample t-test. $\mathrm{P}<0.05$ means there is statistical significance in the difference.

\section{RESULTS}

Among the 128 patients, there were 67 males (52.34\%) and 61 females $(47.66 \%)$. They were aged between 30 and 66 , with the mean age of $47.28 \pm 2.57$ years. Their urticaria duration was from 6 months to 4 years, with the mean of $2.13 \pm 0.58$ years.

After 4 weeks of treatment, the level of serum $\lg E$ in the observation group was lower than that in the control group $(p<0.001)$, and the levels of serum $\mathrm{C} 3$ and $\mathrm{C} 4$ in the observation group were higher than those in the control group (both $p<0.001$ ), as shown in Table I. The levels of serum IL-4, IL-17 and IL-33 in the observation group were lower than those in the control group (all $p<0.001$ ), as shown in Table II. The levels of serum MCP-1, RANTES and Eotaxin in the observation group were lower than those in the control group (all $p<0.001$ ), as shown in Table III.

\section{DISCUSSION}

Urticaria is a common allergic skin disease which has intricate etiology and pathogenesis, and is easy to recurrent. 9 About $15 \%$ and $20 \%$ of people have had urticaria at least once in their lifetime. Those that can be recovered in a short term are referred to as acute urticaria, and those that are repeated for several months or more are referred to as chronic urticaria. ${ }^{10}$ Clinically, many patients with chronic urticaria suffer poor treatment effects and this disease has severely affected their life, work and study. Desloratadine citrate disodium can specifically bind to the $\mathrm{H} 1$ receptor with high selectivity to relieve the symptoms associated with chronic urticaria, and it has the advantages of rapid oral absorption, short onset time, long half-life and less adverse reactions. ${ }^{11,12}$ In this study, desloratadine citrate disodium was used for the treatment of chronic urticaria, and its effects on patients were observed from the aspects of immune parameters, inflammatory factors and chemokines.

Urticaria belongs to the type I allergic reaction. It is mediated by $\lg \mathrm{E}$, which is also known as reactive or pro-

Table I: Comparison of the levels of serum immune function indices after 4 weeks of treatment between the two groups.

\begin{tabular}{l|c|c|c|c|c|c|c}
\hline Groups & $\mathrm{n}$ & \multicolumn{2}{|c|}{$\mathrm{IgE}(\mathrm{IU} / \mathrm{mL})$} & \multicolumn{2}{|c|}{$\mathrm{C}$ (g/L) } & \multicolumn{2}{c}{$\mathrm{C}(\mathrm{g} / \mathrm{L})$} \\
\cline { 3 - 7 } & & Mean $\pm \mathrm{SD}$ & $\mathrm{p}$-value & Mean $\pm \mathrm{SD}$ & $\mathrm{p}$-value & Mean $\pm S D$ & $\mathrm{p}$-value \\
\hline Control group & 64 & $164.75 \pm 28.36$ & $<0.001$ & $0.93 \pm 0.20$ & $<0.001$ & $0.31 \pm 0.06$ & $<0.001$ \\
Observation group & 64 & $92.81 \pm 7.13$ & & $1.22 \pm 0.31$ & & $0.40 \pm 0.07$ & \\
\hline
\end{tabular}

Table II: Comparison of the levels of serum inflammatory factors after 4 weeks of treatment between the two groups.

\begin{tabular}{l|c|c|c|c|c|c|c}
\hline Groups & $\mathrm{n}$ & \multicolumn{2}{|c|}{$\mathrm{LL}-4(\mathrm{ng} / \mathrm{L})$} & \multicolumn{2}{|c|}{$\mathrm{IL}-17$ (ng/L) } & \multicolumn{2}{c}{$\mathrm{IL}-33(\mathrm{ng} / \mathrm{L})$} \\
\cline { 3 - 7 } & & Mean \pm SD & p-value & Mean \pm SD & $\mathrm{p}$-value & Mean \pm SD & $\mathrm{p}$-value \\
\hline Control group & 64 & $6.04 \pm 0.58$ & $<0.001$ & $13.66 \pm 1.69$ & $<0.001$ & $115.24 \pm 18.52$ & $<0.001$ \\
Observation group & 64 & $3.02 \pm 0.49$ & & $9.17 \pm 0.60$ & & $94.85 \pm 13.43$ & \\
\hline
\end{tabular}

Table III: Comparison of the levels of serum chemokines after 4 weeks of treatment between the two groups.

\begin{tabular}{l|c|c|c|c|c|c|c}
\hline Groups & $\mathrm{n}$ & \multicolumn{2}{|c|}{$\mathrm{MCP}-1(\mathrm{pg} / \mathrm{mL})$} & \multicolumn{2}{|c|}{ RANTES $(\mu \mathrm{g} / \mathrm{L})$} & \multicolumn{2}{c}{ Eotaxin $(\mu \mathrm{g} / \mathrm{L})$} \\
\cline { 3 - 7 } & & Mean \pm SD & $\mathrm{p}$-value & Mean \pm SD & $\mathrm{p}$-value & Mean \pm SD & $p$-value \\
\hline Control group & 64 & $301.61 \pm 55.80$ & $<0.001$ & $45.49 \pm 5.64$ & $<0.001$ & $60.39 \pm 7.61$ & $<0.001$ \\
Observation group & 64 & $213.78 \pm 29.58$ & & $33.52 \pm 4.84$ & & $48.35 \pm 5.14$ & \\
\hline
\end{tabular}


cell antibody. IgE is present in the serum of everybody. The content of IgE in normal human serum is very low yet stable, but it will obviously increase in people with allergic diseases. Therefore, IgE is an important marker in allergic diseases. ${ }^{13,14}$ The activation of complement C3 and C4 also plays an important role in the biological effects of IgE antibodies and is directly involved in the subsequent cascade reaction. The more severe the allergic reaction, the more $\mathrm{C} 3$ and $\mathrm{C} 4$ are consumed, and its content in the serum is, therefore, reduced. 15 Studies have shown that more severe chronic spontaneous urticaria is characterised by higher production of $\mathrm{C} 3$ and $\mathrm{C} 4$ complements accompanied by parallel changes in CRP concentration. ${ }^{16}$ This study found that after four weeks of treatment, the level of serum IgE in the observation group was lower than that in the control group, and the levels of $\mathrm{C} 3$ and $\mathrm{C} 4$ were higher in the observation group than those in the control group, indicating that desloratadine citrate disodium can effectively relieve the degree of systemic allergic reactions in patients with chronic urticaria, and its efficacy is better than that of loratadine.

The imbalance of Th1/Th2 cytokines is one of the core factors in the development of chronic urticaria. As the disease progresses, the function of Th2 subpopulation predominates, leading to an increase in the secretion of cytokines IL-4, IL-17 and IL-33.17 IL-4 is a typical factor secreted by Th2 cells, and it can induce the conversion of $\operatorname{lgM}$ to $\operatorname{lgE}$ and aggravate allergic reactions. ${ }^{18}$ Previous studies showed that IL-4 was highly expressed in serum of spontaneous urticaria patients. ${ }^{19} \mathrm{IL}-17$ and IL-33 are newly discovered members of the IL- 1 family. They can regulate immune responses, positively feedback to Th2 cells, and accelerate the activation of Th2 cells and the release of related cytokines. They have been shown to play an important role in most immediate allergic reactions. 20 This study found that the levels of serum IL-4, IL-17 and IL-33 in the observation group were lower than those in the control group after 4 weeks of treatment, suggesting that desloratadine citrate disodium can balance the function of Th1/Th2 cytokines and reduce body allergies more effectively. This conclusion is basically consistent with other studies. 21

In addition to stimulating the body to produce $\lg E$ as allergen and further binding to surface receptors such as mast cells, chemokines also play an important role in the pathogenesis of chronic urticaria. MCP-1 and RANTES are CC-type chemokines that are closely related to allergic reactions and can mediate the production of eosinophils and lymphocyte immunity in the body. The expression levels of MCP-1 and RANTES in patients with allergies are increased to varying degrees. ${ }^{22}$ Belonging to the group $B$ chemokines, Eotaxin induces allergic inflammation and participates in the process of allergic skin damage by regulating eosinophils and Th2 cells to accumulate in inflammation sites. ${ }^{23}$
This study found that the levels of serum MCP-1, RANTES and Eotaxin in the observation group were lower than those in the control group after 4 weeks of treatment, revealing that desloratadine citrate disodium can effectively inhibit the sensitisation of chemokines. This may also be an important mechanism for desloratadine citrate disodium to reduce the severity of chronic urticaria in patients.

There are some limitations to this study. The sample size was small and side effects were not compared. Further research can be done to observe the risk of adverse drug reactions in a large sample of patients with chronic urticaria.

\section{CONCLUSION}

Desloratadine citrate disodium can effectively reduce the severity of chronic urticaria, and its therapeutic mechanism may be related to balancing Th1/Th2 cell function, regulating inflammatory mediators and inhibiting the action of chemokines.

\section{REFERENCES}

1. O'Donnell BF, Lawlor F, Simpson J, Morgan M, Greaves MW. The impact of chronic urticaria on the quality of life. $\mathrm{Br} J$ Dermatol 1997; 136:197-201.

2. Zhou $Y$, Sheng $M$, Chen $M$. Detection and allergen analysis of serum IgE in pediatric patients with chronic urticaria. Pak $J$ Med Sci 2018; 34:385-9.

3. Aamir IS, Tauheed S, Majid F, Atif A. Frequency of autoimmune thyroid disease in chronic urticaria. J Coll Physicians Surg Pak 2010; 20:158-61.

4. Antia C, Baquerizo K, Korman A, Alikhan A, Bernstein JA. Urticaria: A comprehensive review: Treatment of chronic urticaria, special populations, and disease outcomes. J Am Acad Dermatol 2018; 79:617-33.

5. Grattan CE, Dawn G, Gibbs S, Francis DM. Blood basophil numbers in chronic ordinary urticaria and healthy controls: diurnal variation, influence of loratadine and prednisolone and relationship to disease activity. Clin Exp Allergy 2010; 33: 337-41.

6. Basak PY, Vural H, Kazanoglu OO, Erturan I, Buyukbayram HI. Effects of loratadine and cetirizine on serum levels of neuropeptides in patients with chronic urticaria. Int J Dermatol 2015; 53:1526-30.

7. Yuan X, Ghosh A, Jie Q, He G, Wu Y. Effects of desloratadine citrate disodium injection on rat models of ovalbumin-induced allergic rhinitis: involvement of T-cell responses modulation. Int Forum Allergy Rhinol 2016; 5:1170-6.

8. Jie Q, Kodithuwakku ND, Yuan X, He G, Chen M, Xu S, et al. Anti-allergic and anti-inflammatory properties of a potent histamine $\mathrm{H} 1$ receptor antagonist, desloratadine citrate disodium injection, and its anti-inflammatory mechanism on EA.hy926 endothelial cells. Eur J Pharmacol 2015; 754:1-10.

9. Kaplan AP, Joseph K, Maykut RJ, Geba GP, Zeldin RK. Treatment of chronic autoimmune urticaria with omalizumab. J Allergy Clin Immunol 2008; 122:569-73. 
10. Mullol J, Bousquet J, Bachert C, Canonica WG, GimenezArnau A, Kowalski ML, et al. Rupatadine in allergic rhinitis and chronic urticaria. Allergy 2008; 63:5-28.

11. Chen M, Xu S, Zhou P, He G, Jie Q, Wu Y. Desloratadine citrate disodium injection, a potent histamine $H(1)$ receptor antagonist, inhibits chemokine production in ovalbumininduced allergic rhinitis guinea pig model and histamineinduced human nasal epithelial cells via inhibiting the ERK1/2 and NF-kappa B signal cascades. Eur J Pharmacol 2015, 767:98-107.

12. Dong D, Xiao-Liang HE, Yang DY, Wang XX. Comparison of the efficacy of desloratadine citrate disodium and acrivastine decremental therapy in the treatment of chronic urticaria. China Pharm 2015; 26:5078-80.

13. Frosch PJ, Wahl R, Bahmer FA, Maasch HJ. Contact urticaria to rubber gloves is IgE-mediated. Contact Dermatitis 1986; 14:241-5.

14. Wrangsjö K, Wahlberg JE, Axelsson IG. IgE-mediated allergy to natural rubber in 30 patients with contact urticaria. Contact Dermatitis 1988; 19:264-71.

15. Chan MK, Chan KW, Jones B. Immunoglobulins (IgG, IgA, IgM, $\mathrm{IgE}$ ) and complement components (C3, C4) in nephrotic syndrome due to minimal change and other forms of glomerulonephritis, a clue for steroid therapy? Nephron 1987; 47:125-30.

16. Kasperska-Zajac A, Grzanka A, Machura E, Misiolek M, Mazur B, Jochem J. Increased serum complement C3 and C4 concentrations and their relation to severity of chronic spontaneous urticaria and CRP concentration. $J$ Inflamm (Lond) 2013; 10:22.

17. Atwa MA, Emara AS, Youssef N, Bayoumy NM. Serum concentration of IL-17, IL-23 and TNF- $\alpha$ among patients with chronic spontaneous urticaria: association with disease activity and autologous serum skin test. J Eur Acad Dermatol Venereol 2014; 28:469-74.

18. Confino-Cohen R, Goldberg A, Aharoni D, Naiman L, Buchs A, Weiss $\mathrm{M}$, et al. Low stimulated IL-4 secretion in pbmc from patients with chronic idiopathic urticaria. Cytokine 2004; 27:74-80.

19. Czarnecka-Operacz M, Szulczynska-Gabor J, Lesniewska K, Teresiak-Mikolajczak E, Bartkiewicz P, Jenerowicz D, et al. Acute-phase response and its biomarkers in acute and chronic urticaria. Postepy Dermatol Alergol 2018; 35:400-7.

20. Novalrivas M, Burton O, Wise P, Charbonnier LM, Georgiev P, Oettgen $\mathrm{H}$, et al. Regulatory $\mathrm{T}$ cell reprogramming toward a Th2-cell-like lineage impairs oral tolerance and promotes food allergy. Immunity 2015; 42:512-23.

21. Zheng D, Yang X. Clinical observation on the therapeutic effect of desloratadine citrate disodium in the treatment of chronic urticaria and changes in IL4, IL18, IL23 and IL-33 levels before and after treatment. Pak J Pharm Sci 2017; 30:1139-42.

22. Mahalingam S, Karupiah G. Chemokines and chemokine receptors in infectious diseases. Immunol Cell Biol 1999; 77:469-75.

23. Crump MP, Rajarathnam K, Kim KS, Clark-Lewis I, Sykes BD. Solution structure of eotaxin, a chemokine that selectively recruits eosinophils in allergic inflammation. J Biol Chem 1998; 273:22471-9. 